\title{
Home screening for sexually transmitted diseases in high-risk young women: randomised controlled trial
}

\section{Robert L Cook, Lars Østergaard, Sharon L Hillier, Pamela J Murray, Chung-Chou H Chang, Diane M Comer, Roberta B Ness, for the DAISY study team}

See end of article for authors' affiliations

Correspondence to:

Dr R L Cook, Department of Epidemiology and Biostatistics and Medicine University of Florida, Box 100231, Gainesville, FL 32610 , Florida USÁ; cookr@phhp.ufl.edu

Accepted 24 January 2007

\begin{abstract}
Objective: Home screening tests could eliminate several barriers to testing sexually transmitted diseases (STDs).

Aim: To determine whether offering repeated home screening tests would increase the rate of testing for chlamydia and gonorrhoea in a high-risk sample of young women.

Methods: In this randomised controlled trial, 403 young women (mean age 18.9 years, $70 \%$ black) with a recent STD or with STD-related risk factors were enrolled. Participants were recruited from clinics and highprevalence neighbourhoods and then randomly assigned to receive either a home testing kit or an invitation to attend a medical clinic for testing at 6, 12 and 18 months after enrollment. Over $80 \%$ of women were followed for 2 years. The trial is registered with ClinicalTrials.gov, number NCT 00177437.

Results: Of 197 women in the intervention group, 140 (71\%) returned at least one home test and 25 of 249 (10\%) home tests were positive. Women who received home screening tests completed significantly more STD tests overall (1.94 vs 1.41 tests per woman-year, $p<0.001$ ) and more STD tests in the absence of symptoms ( 1.18 vs 0.75 tests per woman-year, $p<0.001)$. More women in the intervention group completed at least one test when asymptomatic (162 (82.2\%) vs $117(61.3 \%), p<0.001)$. The intervention was most effective among women recruited outside medical clinics. There was no significant difference in the overall rate of STDs detected.
\end{abstract}

Conclusions: Home screening significantly increased the utilisation of chlamydia and gonorrhoea testing in this sample of high-risk young women, and thus represents a feasible strategy to facilitate STD testing in young women.

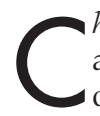
hlamydia trachomatis and Neisseria gonorrhoeae infections are the most common bacterial sexually transmitted diseases (STDs) worldwide. ${ }^{12}$ The majority of infections in women are asymptomatic, and screening young women for chlamydia has been shown to be a cost-effective method of preventing pelvic inflammatory disease. ${ }^{34}$ Therefore, public health organisations in industrialised/developed nations recommend routine screening of sexually active young women for chlamydial infection. ${ }^{5-8}$ Guidelines also suggest screening sexually active women for gonorrhoea infection if they have individual or population risk factors. ${ }^{9}$

Most guidelines recommend annual screening for chlamydia, although more frequent screening has been recommended for some populations. Repeat testing for chlamydia and gonorrhoea 3 months after an infection is now recommended by the Centers for Disease Control and Prevention. ${ }^{5}$ Despite these recommendations, only $33-60 \%$ of at-risk women in the US undergo annual screening. ${ }^{10}{ }^{11}$ A recent report from the National Center for Prevention Priorities listed chlamydia screening as one of the four most important prevention services that is underutilised. ${ }^{12}$ Thus, interventions are needed to increase the rate of screening, especially among women recently diagnosed with an STD and among high-risk women who are not regular users of health clinics.

Home screening tests, using urine samples or self-collected vaginal swabs, have excellent sensitivity and specificity for chlamydia and gonorrhoea detection, with sensitivities generally $>90 \%$ and specificities $>99 \% .^{13}{ }^{14}$ Home testing might overcome several barriers to screening, including individual factors such as privacy, embarrassment or discomfort, and access barriers such as clinic inaccessibility and the time or financial resources needed to attend appointments. In a randomised study of home testing for STDs in Denmark, highschool students who received a single home test were substantially more likely to complete chlamydial screening compared with students who were referred to a clinic for testing. ${ }^{415}$ However, that population had a relatively low risk of infections.

We hypothesised that women at high risk for STDs who received serial home screening tests, compared with women who received serial invitations to attend a clinic for screening, would complete a greater number of chlamydial and gonococcal tests overall, and would be more likely to receive an asymptomatic screening test.

\section{METHODS}

Participants in the DAISY (Detection Acceptability Intervention for STDs in Youth) study were sexually active young women (age 15-24 years) representing two groups of women who might benefit from increased STD testing. First, we recruited women recently diagnosed with an STD (chlamydial, gonococcal or trichomonal infection) because such women are at especially high risk of future STDs and in whom more frequent testing is recommended. ${ }^{5}$ We identified women with a new STD through collaborations with clinicians at 11 community-based medical clinics in Western Pennsylvania. At the majority of these clinics, young women could receive reproductive health services (including STD testing) at minimal or no cost.

A second recruitment effort targeted women in the same neighbourhoods as the clinics, but who were less frequent users of healthcare services (no more than one gynaecological-related visit in the previous year). These participants contacted our

Abbreviations: DAISY, Detection Acceptability Intervention for SIDs in Youth 
research staff in response to advertisements in communities, on public transportation and by referral from other participants. In this second wave of recruitment, women needed to meet at least three of the following five criteria associated with an increased risk of acquiring a STD: young age ( $\leqslant 20$ years), black race, monthly douching, $>1$ sexual partner in the past year or living in a neighbourhood that has within the top 33\% of chlamydial rates in the county. Compared with women recruited directly from neighbourhoods, women recruited directly from clinics were slightly younger (mean age 18.4 vs 19.3 years, $\mathrm{p}=0.0006)$, more likely to be black ( $77 \%$ vs $64 \%$ ), less likely to always use condoms ( $18 \%$ vs $31 \%$ ) and had more sexual partners in the past year (mean 2.9 vs 2.2). However, assignment to intervention or control was nearly identical in the clinic recruits and the neighbourhood recruits.

The study was approved by the institutional review boards at participating institutions and clinics. Recruitment occurred between November 2000 and April 2003. All participants provided written informed consent; we obtained a waiver of the requirement for parental consent, allowing minor participants to sign their own consent forms.

\section{Procedures}

Each woman completed a baseline questionnaire by telephone or during a face-to-face interview with a study research assistant. The baseline questionnaire included an assessment of sociodemographic characteristics, current and past sexual behaviours, and history of STD infection. Women recruited from community neighbourhoods were asked to provide a home test specimen for baseline testing for chlamydia and gonorrhoea. Of the 169 (82\%) women who provided a baseline test, $17 \%$ were positive and treated at baseline.

Women were randomly assigned to one of the two study arms using a computer-generated, blocked randomisation sequence that was delivered in sealed envelopes. Study participants were aware of their randomisation assignment; however, clinicians working in participating clinics and research assistants who abstracted clinical information were blinded to the participants' study assignment.

Women assigned to the intervention group (home testing) received a home testing kit at 6,12 and 18 months after enrollment. Most $(n=179)$ women received their home test in the mail, while 18 picked up their home test at their assigned clinic. The home testing kit materials included a cover letter, an instruction sheet, a brief questionnaire, Dacron-tipped swab, pre-labelled swab container and postage-paid mailing carton. Participants mailed the samples directly to a single-study laboratory.

Vaginal swabs from the home tests were assayed using a nucleic acid amplification test (BD ProbeTec, Becton Dickinson, Sparks, Maryland, USA). In our laboratory, vaginal swab samples have been shown to have a sensitivity and a specificity of 91.9 and 99.8, respectively, for chlamydia, and 100 and 99.8, respectively, for gonorrhoea. ${ }^{16}$ We further assessed home test validity by comparing the results from 97 participants who attended a clinic and had two swabs obtained; one was a vaginal swab that was mailed to the study laboratory in the home test packaging, and the other was a cervical swab sent to the clinic's usual laboratory. The results of these swabs were identical for 95 of 97 swabs tested for chlamydia $(\kappa=0.93)$ and for 88 of 92 tests for gonorrhoea $(\kappa=0.88)$.

Women assigned to the control group received a postcard at 6, 12 and 18 months after enrollment. The postcard included information similar to the cover letter provided with the home test kits, and participants were invited to attend one of the participating study clinics for a routine test for women's health infections at no cost. In neither arm did women receive any incentive or follow-up contact to encourage compliance.
All women were informed that they should maintain their usual care patterns during the follow-up period, including any evaluation for genital symptoms or for STD testing. All women with positive home tests were notified of their test result and counselled about partner notification and referred to one of the participating clinics to receive treatment at no cost. The study investigators occasionally provided treatment in order to facilitate timely treatment for study participants.

All participants were contacted at 1 year and 2 years after enrollment and asked to identify the settings of any gynaecological examinations received since enrollment. Follow-up success was similar in the two groups: $99 \%$ of participants provided data for at least 1 year and $84.5 \%$ completed the 2year follow-up survey. Trained research assistants abstracted medical records from all visits in which a participant received a gynaecological examination or an STD test. Information was recorded regarding symptoms of vaginal discharge or abdominal/pelvic pain, the clinicians' diagnosis, and chlamydial and gonococcal test results. We were able to obtain all known medical records from $90.7 \%$ of women in the home testing group and from $87.4 \%$ of women in the clinic group. There was no significant difference in women with or without missing medical records in terms of age, race, randomisation group, condom use or number of sexual partners in the past year. However, a greater proportion of women with missing medical records were recruited from neighbourhoods rather than from health clinics ( $75 \%$ vs $46 \%$, p $=0.006$ ).

A woman was classified as having had an STD test if a home test was received in our laboratory or if a chlamydial or gonococcal test was documented in a participant's medical record. Tests were classified as symptomatic if the participant reported symptoms of discharge or abdominal pain, and asymptomatic otherwise.

\section{Statistical analysis and sample size calculation}

All analyses used an intention-to-treat approach. The sample size was chosen as 400 in order to have sufficient power $(0.8)$ to determine at least a $30 \%$ increase in the proportion of women who completed at least one asymptomatic screening test per year or in the proportion who acquired a new STD, assuming a $15 \%$ loss to follow-up and $\alpha$ of 0.05 . Of 403 women who provided informed consent, 15 ( 8 in the intervention group and 7 in the control group) were excluded from final analysis because they were lost to follow-up immediately after baseline and never provided any additional follow-up data (fig 1). Inclusion of these women in the analyses did not significantly alter the study conclusions. For the 388 women included in the final analyses, we created count measures to determine the total number of tests, asymptomatic tests, infections detected and infections detected through asymptomatic testing for each participant. We compared baseline characteristics between study groups using $\chi^{2}$ analyses for categorical data and tests for continuous data.

Follow-up for each subject ended at the time of the final STD test or 2-year interview. If a participant did not complete the 2year interview, her data were censored at exactly 1 year (if $<$ l year of follow-up) or 2 years' after her enrollment. We calculated incidence rates and incidence rate ratios for the number of STD tests and the number of STDs detected, and compared these outcomes using the two-sided exact test. We repeated the analyses in subgroups of women, according to whether they were recruited from clinics or from community neighbourhoods.

\section{RESULTS}

At baseline, the mean age of all study subjects was 18.9 years (range 15-24); 70.5\% were black and $90.2 \%$ were not living 


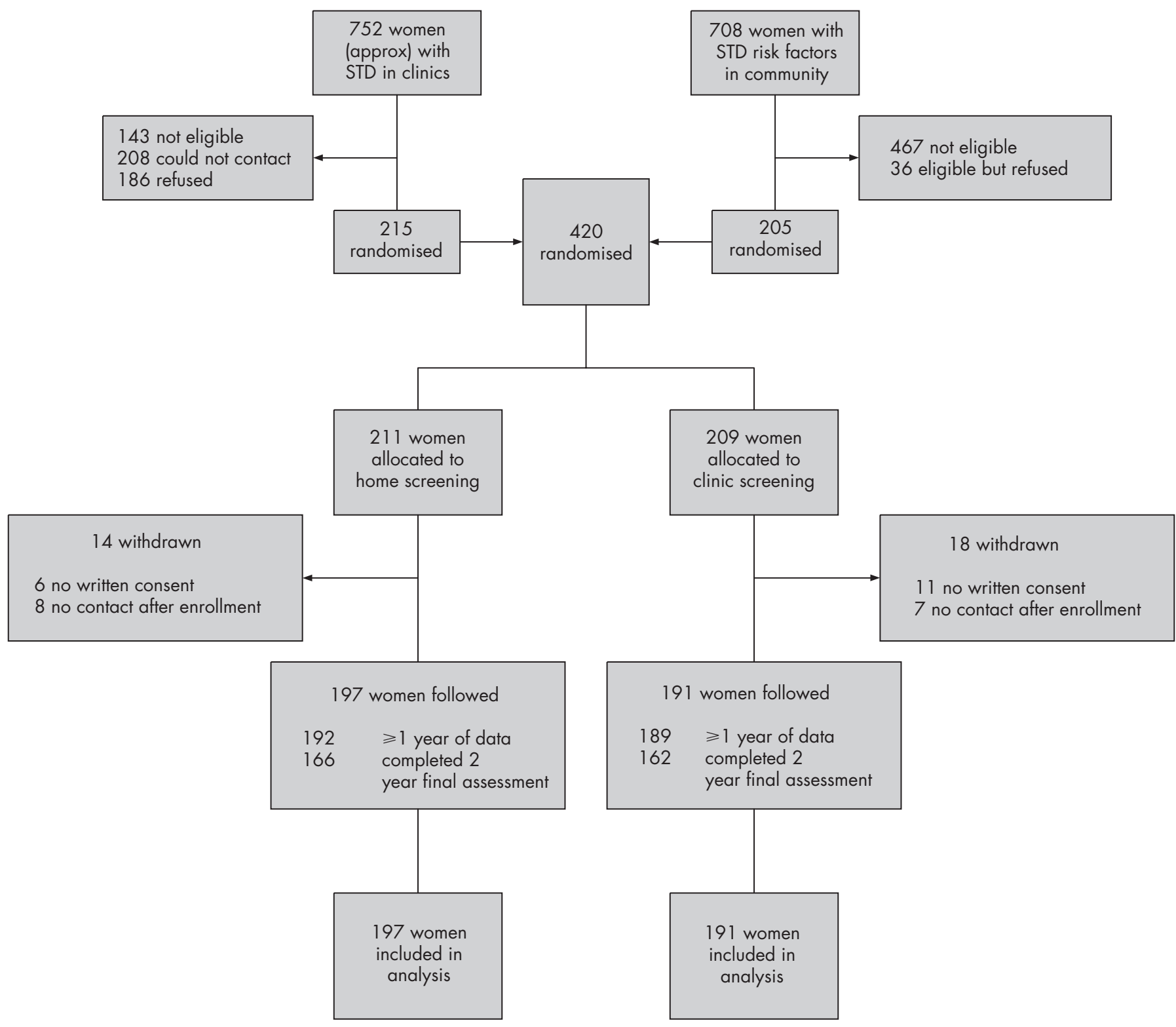

Figure 1 Flowchart of participants in the DAISY (Detection Acceptability Intervention for STDs in Youth).

with a husband or a long-term partner (table 1). There were no significant differences by randomisation group in sexual behaviour during the past year, STD history or contraceptive use. During follow-up, there was no significant difference by intervention group in the proportion of women who reported regular use of condoms at 1-year or 2-year follow-up, or in the proportion of women with a documented Pap smear during the 2 years of follow-up (data not shown).

Of the 249 home tests that were received in the laboratory and interpretable, $25(10.0 \%)$ were positive for chlamydia $(\mathrm{n}=20)$ and/or gonorrhoea $(\mathrm{n}=8)$. All women with a positive home test result were contacted and referred for treatment or treated by study personnel. Most women (71\%) returned at least one home test, whereas $40 \%$ returned two tests and $18 \%$ returned all three home tests.

\section{Number of STD tests completed.}

The number of chlamydia and gonorrhoea tests completed per year was significantly greater in women in the intervention group for all tests ( 1.94 vs 1.41 tests per woman-year, $\mathrm{p}<0.001$ ) and for asymptomatic tests ( 1.18 vs 0.75 tests per woman-year, $\mathrm{p}<0.001$, table 2). The intervention effect appeared to be greatest among women recruited from neighbourhoods, where women in the intervention group were over two times as likely to complete an STD test when asymptomatic or otherwise. The difference in tests completed was largely due to the opportunity for home testing, as the actual number of tests completed in clinics was similar in the two groups.

The proportion of women who completed at least one asymptomatic (screening) test during the 2 years' of followup was significantly greater among women in the intervention group $(82.2 \%$ vs $61.3 \%, \mathrm{p}<0.001$; fig 2$)$. Similarly, the proportion of women who completed $\geqslant 2$ asymptomatic tests was significantly greater among women in the intervention group (55.9\% vs $37.2 \%, p<0.001$; fig 2 ). These differences also seemed to be greater among women recruited from neighbourhoods, compared with those recruited in clinics (data not shown).

\section{Number of STDs detected}

There was no significant difference in the rate of incidence of STDs detected during follow-up in the intervention group 
Table 1 Baseline characteristics of women in the DAISY trial

\begin{tabular}{|c|c|c|c|}
\hline & $\begin{array}{l}\text { Intervention (home screening, } \\
n=197 \text { ) }\end{array}$ & $\begin{array}{l}\text { Control (no home screening, } \\
n=191 \text { ) }\end{array}$ & p Value \\
\hline Age (years), mean (SE) & $19.0(2.6)$ & $18.7(2.9)$ & 0.27 \\
\hline Age dichotomised (years), n (\%) & & & 0.16 \\
\hline $14-18$ & $85(43.1)$ & $96(50.3)$ & \\
\hline 19-25 & $112(56.9)$ & $95(49.7)$ & \\
\hline Race, $n(\%)$ & & & 0.89 \\
\hline Black & $138(70.1)$ & $136(71.2)$ & \\
\hline White & $42(21.3)$ & $41(21.5)$ & \\
\hline Other & $17(8.6)$ & $14(7.3)$ & \\
\hline DAISY recruitment site, $\mathrm{n}(\%)$ & & & 0.99 \\
\hline Adolescent clinic & $42(21.3)$ & $42(22.0)$ & \\
\hline Gynaelogical clinics & $39(19.8)$ & $41(21.5)$ & \\
\hline Student health clinic & $7(3.6)$ & $7(3.7)$ & \\
\hline Women's health or emergency room & $11(5.6)$ & $9(4.7)$ & \\
\hline Community/neighbourhood recruitment & $98(49.8)$ & $92(48.2)$ & \\
\hline \multicolumn{4}{|l|}{ Living situation*, n (\%) } \\
\hline Lives with at least one parent & $85(43.2)$ & $82(42.9)$ & 0.69 \\
\hline Lives with own child (children) & $60(30.5)$ & $47(24.6)$ & 0.31 \\
\hline Lives with husband/long-term partner & $14(7.1)$ & $21(11.0)$ & 0.39 \\
\hline Not a high-school graduate, n (\%) & $82(41.6)$ & $91(47.6)$ & 0.23 \\
\hline Uninsured or on medical assistance, $\mathrm{n}(\%)$ & $135(68.5)$ & $124(64.9)$ & 0.45 \\
\hline Number of partners during the past year, mean (SE) & $2.6(3.5)$ & $2.4(1.5)$ & 0.46 \\
\hline Exchange sex for money, drugs, and so on in the past year, $n(\%)$ & $0(0.00)$ & $4(2.1)$ & 0.06 \\
\hline \multicolumn{4}{|l|}{ Contraceptive method used in past 6 months, ${ }^{*} \mathrm{n}(\%)$} \\
\hline None & 25 (12.7) & $29(15.2)$ & 0.48 \\
\hline Birth control pills & $64(32.5)$ & $54(28.3)$ & 0.37 \\
\hline Condoms (any) & $162(82.2)$ & $154(80.6)$ & 0.68 \\
\hline Spermicide & 33 (16.8) & $25(13.1)$ & 0.31 \\
\hline Depo provera shot & $33(16.8)$ & 37 (19.4) & 0.50 \\
\hline Condom use (always), $\mathrm{n}(\%)$ & $51(25.9)$ & $44(23.0)$ & 0.51 \\
\hline
\end{tabular}

compared with the control group (20.4 vs 24.1 infections per 100 woman-years, $\mathrm{p}=0.28)$. The results were similar when restricted to chlamydia only (17.6 vs 18.9 infections per 100 woman-years) or when restricted to gonorrhoea only (4.9 vs 7.9 infections per 100 woman-years). There was also no difference in the rate of STDs detected during asymptomatic testing or when the analyses were restricted to those women recruited from clinics (28.7 vs 35.2 infections per 100 woman-years) or to those recruited from the community (11.3 vs 11.2 infections per 100 woman-years).

\section{DISCUSSION}

This randomised controlled trial differs from previous studies of home testing in that it evaluated whether women would use home testing repeatedly over time, as opposed to accepting a single test. The study included women at especially high risk for STDs and those who had excellent follow-up rates over 2 years. We found that women who received a home testing intervention completed significantly more STD tests overall and more
STD tests when asymptomatic, compared with women who did not receive the home testing option. The proportion of women who completed at least one asymptomatic test during follow-up was also significantly greater among women who received home tests. Thus, home screening provides a clear option to increase the rate of recommended screening for chlamydial and gonococcal infections in high-risk women, especially in women who are not regular users of the healthcare system.

Home screening for STDs has been evaluated in a variety of public health situations, including mass mailings to general population samples, college students and patient samples ${ }^{17-22}$; the placement of home testing kits in a neighbourhood pharmacy and a gymnasium ${ }^{23}$; facilitation of repeat testing after a positive infection ${ }^{24}{ }^{25}$; testing of male partners of women who had been diagnosed with chlamydia infection ${ }^{26}$; and from online STD testing sites. ${ }^{27}{ }^{28}$ In another randomised controlled trial of home screening, Danish students who received a single home screening test completed a significantly greater number of screening tests, had a greater number of infections detected,

Table 2 Young women's rates of testing for chlamydia or gonorrhoea during 2 years' of follow-up, according to whether they received home screening tests

\begin{tabular}{|c|c|c|c|}
\hline & \multicolumn{2}{|c|}{ STD tests completed per year } & \multirow[b]{2}{*}{ Rate ratio $(95 \% \mathrm{Cl})$} \\
\hline & $\begin{array}{l}\text { Home screening } \\
(\mathrm{n}=197)\end{array}$ & $\begin{array}{l}\text { No home screening } \\
(n=191)\end{array}$ & \\
\hline \multicolumn{4}{|l|}{ All participants } \\
\hline All tests & 1.94 & 1.41 & 1.38 (1.23 to 1.55$)$ \\
\hline Asymptomatic tests & 1.18 & 0.75 & 1.57 (1.34 to 1.83$)$ \\
\hline \multicolumn{4}{|c|}{ Women recruited from neighbourhoods $(n=190)$} \\
\hline All tests & 1.47 & 0.69 & $2.12(1.70$ to 2.66$)$ \\
\hline Asymptomatic tests & 0.98 & 0.44 & $2.22(1.68$ to 2.95$)$ \\
\hline \multicolumn{4}{|c|}{ Women recruited from clinics $(n=198)$} \\
\hline All tests & 2.38 & 2.02 & 1.18 (1.03 to 1.35$)$ \\
\hline Asymptomatic tests & 1.36 & 1.02 & 1.34 (1.11 to 1.62$)$ \\
\hline
\end{tabular}




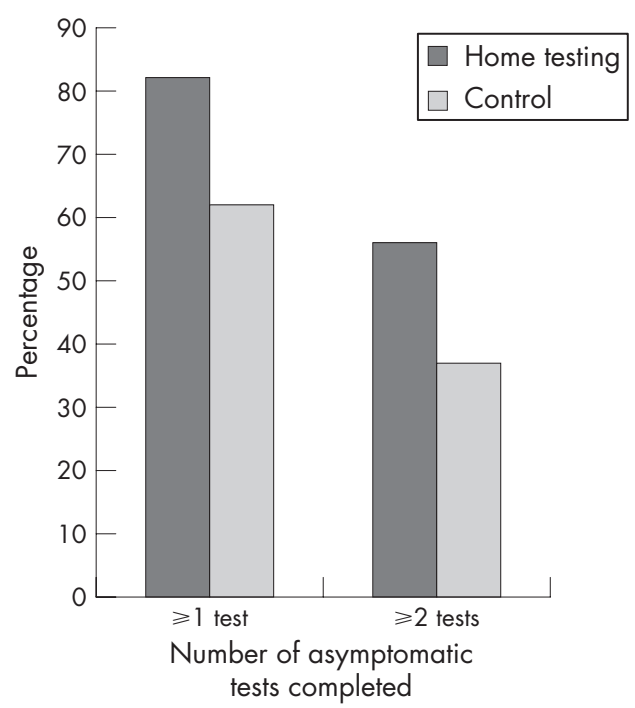

Figure 2 Proportion of women who completed at least one and at least two asymptomatic sexually transmitted disease tests during 2 years' of follow-up. Differences in the two groups were each statistically significant at $\mathrm{p}<0.001$.

and had a lower incidence of pelvic inflammatory disease, compared with those who received an invitation to attend a clinic. ${ }^{4}{ }^{14}$

The potential benefits of home screening could vary in different settings. In our study, the intervention appeared to be most effective in increasing the rate of completion of STD screening tests in women recruited from neighbourhoods (who were less regular users of healthcare services) rather than in women recruited from healthcare clinics (many of whom were frequent users of healthcare services). The women recruited from the neighbourhood had similar access to free reproductive healthcare services and lived in the same neighbourhoods, but could have some other reasons for using clinic services less frequently. Although the characteristics of women recruited from the two samples were somewhat different, each sample was randomised equally to the intervention group and the control group, so it is unlikely that the differences in the samples had a significant impact on the overall results.

A potential consequence of any screening test, including home screening tests, is a false-positive result. Predictive values of home screening tests will vary according to the prevalence of infection in the population being screened. For example, using the average test characteristics of a home chlamydia test $(90 \%$ sensitivity and $99 \%$ specificity), ${ }^{13}{ }^{16}$ the positive predictive value and negative predictive value will be $91 \%$ and $99 \%$, respectively, if the prevalence is $10 \%$ (as in our study population), but will be $73 \%$ and $99 \%$, respectively, if the prevalence is $3 \%$ (a more typical average in the community). Thus, clinicians might consider confirmatory testing of positive home tests in a lower prevalence setting. Finally, it is important to ensure that women who receive home testing for certain STDs do not fail to receive other important reproductive healthcare services such as recommended cervical cancer screening or testing and counselling for HIV and other STDs. In our study, the rates of cervical cancer screening were identical in the intervention and control groups, suggesting that the availability of home screening did not deter women from receiving other reproductive health services.

Interestingly, the increase in testing did not result in a corresponding increase in the rate of STD detection in this population. Because this was a randomised controlled trial, we expected that the rate of STD acquisition would be similar in the two groups, but that increased testing might detect more infections. On the other hand, the finding of similar STD rates in both groups provides reassurance that offering home testing will not lead women to avoid clinics and miss detection of clinically important infections. In our study, the majority of women could receive STD testing and care at no cost at most of the participating clinics, and all participants lived within 15 min of at least one participating clinic. Also, clinicians in participating clinics frequently did STD testing. Thus, the overall added clinical utility of additional home screening in this population with ready access and high utilisation of clinical care remains unproven.

Several study limitations warrant mention. Women who were uncomfortable with home testing might have been more likely to decline participation in the clinical trial; thus, the utilisation of home testing may differ in women who do not participate in research studies. Our definition of STD symptoms was limited to discharge or abdominal pain, which could result in some women with other symptoms being labelled as asymptomatic. Also, we sent out home screening tests at fixed intervals, and it is possible that allowing women to determine their own testing interval (eg, after a new partner contact) could have a different impact.

In summary, we found that home testing using self-collected vaginal swabs can increase STD testing rates among young women at high risk for STDs. Because the intervention appeared to be most effective in women who did not routinely use clinical care, organisations responsible for a large population of young women might consider using home testing to increase screening rates. In other settings, young women consistently report that self-testing is easy to perform and prefer self-testing over routine gynaecological examinations. ${ }^{29} 30$ Furthermore, home testing is likely to cost much less overall compared with an examination in a clinic, which requires an examination room, sterile supplies and a trained clinician to conduct it. Therefore, the results of this study confirm that home testing is a feasible method to improve screening rates for STDs in young women.

\section{ACKNOWLEDGEMENTS}

This study was funded by the US Agency for Healthcare Research and Quality. The DAISY study team included Shari Hutchison, Kathryn Mazzaferro, Allison Gauspari, Jacqueline Washington, Debby Bass and Sherry Kelsey, who assisted with recruitment and data analysis; Kim Rosenwald, Denita Haraburda, JoAnne Smith, Beverlyn Chapple, Kathy

\section{Key messages}

- Home screening tests for chlamydia and gonorrhoea, mailed to young women at high risk for sexually transmitted diseases (STDs), resulted in an increased number of screening tests completed.

- Home screening tests for chlamydia and gonorrhoea seemed to be most effective at increasing screening among young women who were not already frequent users of reproductive health services.

- In this sample of young women, $10 \%$ of home screening tests were positive for chlamydia or gonorrhoea, and all women with positive tests were successfully notified and treated.

- The true clinical impact of home screening tests in highrisk populations remains unclear, as there were no significant differences between women randomised to home screening versus those randomised to clinic testing with respect to the number of STDs detected and treated. 
Klobucher, Mary-Dea Johnston, Charlynn Bailey, Kathy Biddle, Karen Franklin, Patty Lewis, Margie Janusey, Jennifer Hayes and Kathy Guliano, who were involved with clinical care at participating clinics; and Gale Burstein, Delia Scholes and Marie Diener-West, who served on the DSMB and provided advice regarding the study design and outcomes.

\section{Authors' affiliations}

Robert L Cook, Departments of Epidemiology and Biostatistics and Medicine, University of Florida, Gainesville, Florida, USA

Lars Østergaard, Department of Infectious Diseases, Aarhus University Hospital, Aarhus, Denmark

Sharon L Hillier, Roberta B Ness, Department of Obstetrics, Gynecology and Reproductive Health Services, University of Pittsburgh, Pittsburgh, Pennsylvania, USA

Pamela J Murray, Department of Pediatrics, University of Pittsburgh, Pittsburgh, Pennsylvania, USA

Chung-Chou H Chang, Department of Biostatistics, University of Pittsburgh, Pittsburgh, Pennsylvania, USA

Roberta B Ness, Department of Epidemiology, University of Pittsburgh, Pittsburgh, Pennsylvania, USA

Chung-Chou H Chang, Diane M Comer, The Center for Research on Health Care, University of Pittsburgh, Pittsburgh Pennsylvania, USA

Competing interests: None declared.

Contributors: RLC participated in the design of the study, monitoring of procedures, data analysis, interpretation of findings and writing of the manuscript. LØ participated in the design of the study, interpretation of findings and writing of the manuscript. SLH participated in the design of the study and overseeing of laboratory issues involving home testing. PJM participated in the design of the study, interpretation of findings and writing of the manuscript. CCC conducted data analyses and provided statistical advice. DMC conducted data analyses and contributed to the writing of the manuscript. RBN participated in the design of the study, monitoring of procedures, data analyses, interpretation of findings and writing of the manuscript.

\section{REFERENCES}

1 World Health Organization. Global prevalence and incidence of selected curable sexually transmitted diseases: overview and estimates. Geneva: WHO, 2001.

2 Centers for Disease Control and Prevention. Sexually transmitted disease surveillance 2004. Atlanta, GA: US, Department of Health and Human Services, Centers for Disease Control and Prevention, 2005.

3 Scholes D, Stergachis A, Heidrich F, et al. Prevention of pelvic inflammatory disease by screening for cervical chlamydial infection. N Engl J Med 1996;334:1362-6.

4 Ostergaard L, Andersen B, Moller JK, et al. Home sampling versus conventional swab sampling for screening of Chlamydia trachomatis in women: a clusterrandomized 1-year follow-up study. Clin Infect Dis 2000;31:951-7.

5 Centers for Disease Control and Prevention. Sexually transmitted diseases treatment guidelines 2006. MMWR Recomm Rep, 2006;55(No.RR-11).

6 Hollblad-Fadiman K, Goldman SM. American College of Preventive Medicine practice policy statement: screening for Chlamydia trachomatis. Am J Prev Med 2003;24:287-92.

7 Nelson HD, Helfand M. Screening for chlamydial infection. Am J Prev Med $2001 ; 20$ (Suppl):95-107.

8 Scottish Intercollegiate Guidelines Network. Management of genital Chlamydia trachomatis infection: a national clinical guideline (SIGN publication number 42). Edinburgh: SIGN, 2000.
9 US Preventive Services Task Force. Screening for gonorrhea: recommendation statement. Ann Fam Med 2005;3:263-7.

10 Cook RL, Wiesenfeld HC, Ashton MR, et al. Barriers to screening sexually active adolescent women for chlamydia: a survey of primary care physicians. J Adolesc Health 2001;28:204-10.

11 Levine WC, Dicker LW, Devine O, et al. Indirect estimation of Chlamydia screening coverage using public health surveillance data. Am J Epidemiol 2004;160:91-6.

12 Maciosek MV, Coffield AB, Edwards NM, et al. Priorities among effective clinical preventive services: results of a systematic review and analysis. Am J Prev Med 2006;31:52-61.

13 Cook R, Hutchison S, Ostergaard L, et al. Noninvasive testing for chlamydia and gonorrhea: a systematic review. Ann Intern Med 2005;142:914-25.

14 Schachter J, Chernesky MA, Willis DE, et al. Vaginal swabs are the specimens of choice when screening for Chlamydia trachomatis and Neisseria gonorrhoeae: results from a multicenter evaluation of the APTIMA assays for both infections. Sex Transm Dis 2005;32:725-8.

15 Ostergaard L, Andersen B, Olesen F, et al. Efficacy of home sampling for screening of Chlamydia trachomatis: randomised study. BMJ 1998;317:26-7.

16 Cosentino LA, Landers DV, Hillier SL. Detection of Chlamydia trachomatis and Neisseria gonorrhoeae by strand displacement amplification and relevance of the amplification control for use with vaginal swab specimens. J Clin Microbiol 2003:41:3592-6.

17 van Bergen J, Gotz HM, Richardus JH, et al. Prevalence of urogenital Chlamydia trachomatis increases significantly with level of urbanisation and suggests targeted screening approaches: results from the first national population based study in the Netherlands. Sex Transm Infect 2005;81:17-23.

18 Macleod J, Salisbury C, Low N, et al. Coverage and uptake of systematic postal screening for genital Chlamydia trachomatis and prevalence of infection in the United Kingdom general population: cross sectional study. BMJ 2005;330:940.

19 Andersen B, Olesen F, Moller JK, et al. Population-based strategies for outreach screening of urogenital Chlamydia trachomatis infections: a randomized, controlled trial. J Infect Dis 2002; 185:252-8.

20 Rogstad KE, Bates SM, Partridge S, et al. The prevalence of Chlamydia trachomatis infection in male undergraduates: a postal survey. Sex Transm Infect 2001;77:111-13.

21 Stephenson J, Carder C, Copas A, et al. Home screening for chlamydial genital infection: is it acceptable to young men and women? Sex Transm Infect 2000;76:25-7.

22 van Valkengoed IG, Morre SA, van den Brule AJ, et al. Low diagnostic accuracy of selective screening criteria for asymptomatic Chlamydia trachomatis infections in the general population. Sex Transm Infect 2000;76:375-80.

23 Bloomfield PJ, Kent C, Campbell D, et al. Community-based chlamydia and gonorrhea screening through the United States mail, San Francisco. Sex Transm Dis 2002;29:294-7.

24 Bloomfield PJ, Steiner KC, Kent CK, et al. Repeat chlamydia screening by mail, San Francisco. Sex Transm Infect 2003;79:28-30.

25 Kjaer HO, Dimcevski G, Hoff G, et al. Recurrence of urogenital Chlamydia trachomatis infection evaluated by mailed samples obtained at home: 24 weeks' prospective follow up study. Sex Transm Infect 2000;76:169-72.

26 Andersen B, Ostergaard L, Moller JK, et al. Home sampling versus conventional contact tracing for detecting Chlamydia trachomatis infection in male partners of infected women: randomised study. BMJ 1998;316:350-1.

27 Gaydos CA, Dwyer K, Barnes M, et al. Internet-based screening for Chlamydia trachomatis to reach nonclinic populations with mailed self-administered vaginal swabs. Sex Transm Dis 2006;33:451-7.

28 Novak DP, Karlsson RB. Simplifying chlamydia testing: an innovative Chlamydia trachomatis testing approach using the internet and a home sampling strategy: population based study. Sex Transm Infect 2006;82:142-7.

29 Wiesenfeld HC, Lowry DL, Heine RP, et al. Self-collection of vaginal swabs for the detection of chlamydia, gonorrhea, and trichomoniasis: opportunity to encourage sexually transmitted disease testing among adolescents. Sex Transm Dis $2001 ; 28: 321-5$

30 Chernesky MA, Hook EW III, Martin DH, et al. Women find it easy and prefer to collect their own vaginal swabs to diagnose Chlamydia trachomatis or Neisseria gonorrhoeae infections. Sex Transm Dis 2005;32:729-33. 